\title{
DIFFERENTIATION OF REAL-VALUED FUNCTIONS AND CONTINUITY OF METRIC PROJECTIONS
}

\author{
SIMON FITZPATRICK
}

\begin{abstract}
We characterize the Fréchet differentiability of real-valued functions on certain real Banach spaces in terms of a directional derivative being equal to a modified version of the local Lipschitz constant. This yields the continuity of metric projections onto closed sets whose distance functions have directional derivatives equal to 1 , provided the Banach space and its dual have Fréchet differentiable norms.
\end{abstract}

1. Introduction. Let $E$ be a real Banach space and let $M$ be a closed subset of $E$. We define the distance function

$$
\varphi_{M}(x)=\inf \{\|y-x\|: y \in M\}
$$

and the metric projection

$$
P_{M}(x)=\left\{y \in M:\|y-x\|=\varphi_{M}(x)\right\},
$$

which assigns to each $x \in E$ the set of nearest points in $M$ to $x$. We call a sequence $\left(y_{n}\right)$ from $M$ a minimizing sequence for $x$ provided $\left\|x-y_{n}\right\| \rightarrow \varphi_{M}(x)$ as $n \rightarrow \infty$ and we say that $P_{M}$ is continuous at $x$ provided $y_{n} \rightarrow y_{0}$ whenever $y_{n} \in P_{M}\left(x_{n}\right)$ for all $n \geq 0$ and $x_{n} \rightarrow x_{0}$. If every minimizing sequence for $x$ converges then $P_{M}$ is continuous at $x$; the converse holds in Banach spaces whose norms are sufficiently well behaved (see [2]).

For a real-valued function $f$ on $E$, a point $x$ of $E$ and $u \in S(E)=\{y \in E:\|y\|=$ 1) define the directional derivative

$$
D_{u} f(x)=\lim _{t \rightarrow 0} t^{-1}[f(x+t u)-f(x)]
$$

if it exists. If the limit in (1.1) exists uniformly for $u \in S(E)$ we say that $f$ is Fréchet differentiable at $x$; that is equivalent to existence of $x^{*} \in E^{*}$ (the Fréchet derivative of $f$ at $x$ ) such that

$$
\lim _{\|y\| \rightarrow 0} \frac{f(x+y)-f(x)-x^{*}(y)}{\|y\|}=0
$$

and we write $f^{\prime}(x)=x^{*}$ and note that $f^{\prime}(x)(u)=D_{u} f(x)$ for $u \in S(E)$.

In $\S 2$ we will show that a real-valued function $f$ is Fréchet differentiable at a point $x$ whenever there exists $u \in S(E)$ such that the norm is Fréchet differentiable at $u$ and $D_{u} f(x)$ equals a number we define and call $N_{f}(x)$; it is dominated by the local Lipschitz constant of $f$ at $x$. For reflexive Banach spaces whose norms are Fréchet differentiable (except at 0 , of course) this characterizes the Fréchet derivative of a real-valued function.

Received by the editors September 20, 1983.

1980 Mathematics Subject Classification. Primary 46G05, 41A65. 
Applying this in $\S 3$ we show that if $D_{u} \varphi_{M}(x)=1$ for some $u \in S(E)$ and the norm of $E$ has Fréchet derivative $u^{*}$ at the point $u$ then $\varphi_{M}$ is Fréchet differentiable at $x$ and $\varphi_{M}^{\prime}(x)=u^{*}$. If, in addition, the norm of $E^{*}$ is Fréchet differentiable at $u^{*}$ then the corresponding metric projection $P_{M}$ is continuous at $x$. This uses and improves on results from [2].

This continuity result when combined with a result of Vlasov [6] shows that if $E$ and $E^{*}$ have Fréchet differentiable norms and $\varphi_{M}$ has a directional derivative equal to 1 at each point outside $M$, then $M$ is convex.

2. Differentiability of real-valued functions. Suppose $f$ is a real-valued function on a Banach space $E$ and $x \in E$. We define the local Lipschitz constant of $f$ at $x$ by

$$
L_{f}(x)=\lim _{\delta \rightarrow 0+} \omega_{f}(x, \delta)
$$

where

$$
\omega_{f}(x, \delta)=\sup \{|f(y)-f(z)| /\|y-z\|:\|y-x\| \leq \delta,\|z-x\| \leq \delta, y \neq z\} .
$$

Similarly we define

$$
N_{f}(x)=\lim _{\delta \rightarrow 0+} \nu_{f}(x, \delta)
$$

where

$$
\nu_{f}(x, \delta)=\sup \{|f(y)-f(z)| /\|y-z\|: 2\|z-x\| \leq\|y-x\| \leq \delta, y \neq z\} .
$$

EXAMPLE. To see that $L_{f}$ and $N_{f}$ are not always equal, consider $f(x)=$ $x^{2} \sin \left(x^{-1}\right)$ for $x \in \mathbf{R} \backslash\{0\}$, taking $f(0)=0$. Then $L_{f}(0)=1$ while $N_{f}(0)=0$ and $f^{\prime}(0)=0$. This suggests that $N_{f}$ has more to do with the derivative than $L_{f}$ has.

We will need the following trivial lemma.

2.1 LEMMA. $N_{f}(x) \leq L_{f}(x)$ and $N_{f}(x)$ is the least number $N$ such that for each $\varepsilon>0$ there is $\delta>0$ with $|f(y)-f(z)| \leq(N+\varepsilon)\|y-z\|$ whenever $2\|z-x\| \leq$ $\|y-x\| \leq \delta$.

Now we show why $N_{f}(x)$ is a good bound on the derivatives.

2.2 THEOREM. (i) If $u \in S(E)$ and $D_{u} f(x)$ exists then $D_{u} f(x) \leq N_{f}(x)$.

(ii) If $f$ is Fréchet differentiable at $x$ then $\left\|f^{\prime}(x)\right\|=N_{f}(x)$.

ProOF. (i) For $t>0$ we have

$$
t^{-1}(f(x+t u)-f(x))=(f(x+t u)-f(x)) /\|t u\| \leq \nu_{f}(x, t),
$$

so in the limit as $t \rightarrow 0+$ we obtain $D_{u} f(x) \leq N_{f}(x)$.

(ii) Since $\left\|f^{\prime}(x)\right\|=\sup \left\{f^{\prime}(x)(u): u \in S(E)\right\}$ and $f^{\prime}(x)(u)=D_{u} f(x) \leq N_{f}(x)$ for all $u \in S(E)$, we have $\left\|f^{\prime}(x)\right\| \leq N_{f}(x)$. Write $x^{*}=f^{\prime}(x)$ and for each $\varepsilon>0$ choose $\delta>0$ such that $\|y-x\|<\delta$ implies that

$$
\left|f(y)-f(x)-x^{*}(y-x)\right| \leq \varepsilon\|y-x\| .
$$

Then for $2\|z-x\| \leq\|y-x\| \leq \delta$ we have

$$
\left|f(y)-f(x)-x^{*}(y-x)-f(z)+f(x)+x^{*}(z-x)\right| \leq \varepsilon\|y-x\|+\varepsilon\|z-x\|
$$

so that

$$
|f(y)-f(z)| \leq\left|x^{*}(y-z)\right|+\varepsilon(\|y-x\|+\|z-x\|) .
$$


Now $\mid x^{*}(y-z) \leq\left\|f^{\prime}(x)\right\| \cdot\|y-z\|$ and $2\|z-x\| \leq\|y-x\|$ implies by the triangle inequality that $\|z-x\| \leq\|y-z\|$ and that $\|y-x\| \leq 2\|y-z\|$. Thus

$$
|f(y)-f(z)| \leq\left\|f^{\prime}(x)\right\| \cdot\|y-z\|+3 \varepsilon\|y-z\|
$$

so that $N_{f}(x) \leq\left\|f^{\prime}(x)\right\|$ by Lemma 2.1 , which completes the proof.

2.3 COROLlaRY. If $E$ is reflexive and $f$ is Fréchet differentiable at $x$ then there is $u \in S(E)$ with $N_{f}(x)=D_{u} f(x)$.

Proof. We have $N_{f}(x)=\left\|f^{\prime}(x)\right\|$ and since $E$ is reflexive there is $u \in S(E)$ such that $f^{\prime}(x)(u)=\left\|f^{\prime}(x)\right\|$. Thus $D_{u} f(x)=f^{\prime}(x)(u)=N_{f}(x)$.

From the definiton we easily see that if $N_{f}(x)=0$ then $f$ is Fréchet differentiable at $x$ and $f^{\prime}(x)=0$. Our main result is a partial converse to Corollary 2.3.

2.4 THEOREM. Let $E$ be a real Banach space and $f$ a real-valued function on $E$. Suppose that $D_{u} f(x)=N_{f}(x)$ for some $x \in E$ and $u \in S(E)$. If the norm of $E$ is Fréchet differentiable at $u$ with derivative $u^{*}$, then $f$ is Fréchet differentiable at $x$ and $f^{\prime}(x)=N_{f}(x) u^{*}$.

PROOF. Let $0<\varepsilon \leq \frac{1}{2}$ and choose $0<\gamma \leq \varepsilon$ such that $\|u+z\|-\|u\| \leq$ $u^{*}(z)+\varepsilon\|z\|$ whenever $\|z\| \leq \gamma$. Now let $\delta>0$ be such that $\mid f(x+t u)-f(x)-$ $t D_{u} f(x)\left|\leq \gamma^{2}\right| t \mid$ whenever $|t| \leq \delta$ and $|f(y)-f(z)| \leq\left(N_{f}(x)+\gamma^{2}\right)\|y-z\|$ whenever $0 \leq 2\|z-x\| \leq\|y-x\| \leq \delta$.

Suppose $0<\|w\| \leq \gamma \delta$ and let $t=\gamma^{-1}\|w\|$. Thus $0<t \leq \delta,\left\|t^{-1} w\right\|=\gamma$ and $2\|w\|=2 \gamma t \leq t=\|t u\|$. Hence $\left\|u \pm t^{-1} w\right\|-\|u\| \leq \pm u^{*}\left(t^{-1} w\right)+\varepsilon\left\|t^{-1} w\right\|$ and $|f(x+w)-f(x \pm t u)| \leq\left(N_{f}(x)+\gamma^{2}\right)\|w \mp t u\|$. Now

$$
\begin{aligned}
f(x+w)-f(x) & =\{f(x+w)-f(x-t u)\}+\{f(x-t u)-f(x)\} \\
& \leq\left(N_{f}(x)+\gamma^{2}\right)\|w+t u\|-D_{u} f(x) t+\gamma^{2} t \\
& =N_{f}(x)(\|w+t u\|-t)+\gamma^{2}(\|w+t u\|+t) \\
& \leq t N_{f}(x)\left(\left\|u+t^{-1} w\right\|-\|u\|\right)+3 \gamma^{2} t \\
& \leq t N_{f}(x)\left(u^{*}\left(t^{-1} w\right)+\varepsilon\left\|t^{-1} w\right\|\right)+3 \gamma^{2} t \\
& \leq N_{f}(x) u^{*}(w)+\varepsilon N_{f}(x)\|w\|+3 \gamma\|w\| \\
& \leq N_{f}(x) u^{*}(w)+\varepsilon\left(N_{f}(x)+3\right)\|w\| .
\end{aligned}
$$

For the reverse inequality

$$
\begin{aligned}
f(x+w)-f(x) & =\{f(x+w)-f(x+t u)\}+\{f(x+t u)-f(x)\} \\
& \geq-\left(N_{f}(x)+\gamma^{2}\right)\|t u-w\|+D_{u} f(x) t-\gamma^{2} t \\
& =-t N_{f}(x)\left(\left\|u-t^{-1} w\right\|-\|u\|\right)-\gamma^{2}(t+\|t u-w\|) \\
& \geq-t N_{f}(x)\left(u^{*}\left(-t^{-1} w\right)+\varepsilon\left\|t^{-1} w\right\|\right)-3 \gamma^{2} t \\
& =N_{f}(x) u^{*}(w)-N_{f}(x) \varepsilon\|w\|-3 \gamma\|w\| \\
& \geq N_{f}(x) u^{*}(w)-\varepsilon\left(N_{f}(x)+3\right)\|w\| .
\end{aligned}
$$

Therefore $\left|f(x+w)-f(x)-N_{f}(x) u^{*}(w)\right| \leq\left(N_{f}(x)+3\right) \varepsilon\|w\|$ whenever $\|w\| \leq \gamma \delta$ and hence $f^{\prime}(x)=N_{f}(x) u^{*}$. 
2.5 CoRollary. Let $E$ be a reflexive Banach space with Fréchet differentiable norm. A real valued function $f$ on $E$ is Fréchet differentiable at a point $x$ if and only if there is $u \in S(E)$ such that $D_{u} f(x)$ exists and equals $N_{f}(x)$.

PROOF. If such a vector $u$ exists then Theorem 2.4 shows that $f^{\prime}(x)$ exists. Conversely if $f^{\prime}(x)$ exists then Corollary 2.3 shows that there is such a vector $u$.

Since $L_{f}(x) \geq N_{f}(x) \geq D_{u} f(x)$ (if this exists) for all $u \in S(E)$ we have the following result.

2.6 COROLlaRY. If $D_{u} f(x)=L_{f}(x)$ for some $x \in E$ and $u \in S(E)$ such that the norm of $E$ is Fréchet differentiable at $u$ with derivative $u^{*}$, then $f$ is Fréchet differentiable at $x$ and $f^{\prime}(x)=L_{f}(x) u^{*}$.

3. Continuity of metric projections. In this section we apply the results of the previous section to the distance function $\varphi=\varphi_{M}$ from a closed subset $M$ of the real Banach space $E$ to get continuity results for the metric projection $P=P_{M}$.

Recall that $u^{*} \in E^{*}$ strongly exposes a subset $C$ of $E$ at a point $z$ of $C$ provided $\left\|y_{n}-z\right\| \rightarrow 0$ whenever $y_{n} \in C$ and $u^{*}\left(y_{n}\right) \rightarrow u^{*}(z)$. The following basic result dates back to Smulian [5]; see also $[3, \S 3.4$ or 4]. Let $B(E)$ denote the closed unit ball of $E$.

3.1 PROPOSITION. The norm of $E^{*}$ is Fréchet differentiable at a point $u^{*}$ of $E^{*}$ if and only if $u^{*}$ strongly exposes $B(E)$. The norm of $E$ is Fréchet differentiable at a point $u$ with derivative $u^{*}$ if and only if $B\left(E^{*}\right)$ is strongly exposed at $u^{*}$ by $u \in E \subseteq E^{* *}$.

We will need the following result from [2].

3.2 PROPOSITION. If $x \in E \backslash M$ is a point of Fréchet differentiability of $\varphi$ then $\left\|\varphi^{\prime}(x)\right\|=1$. If $\varphi^{\prime}(x)$ strongly exposes the unit ball of $E$ at a point $z$ then every minimizing sequence for $x$ converges to $x-\varphi(x) z$.

The following result improves Theorem 2.4(a) of [2].

3.3 TheOREM. Suppose that $x \in E$ and $u \in S(E)$ with $D_{u} \varphi(x)=1$. If $u^{*}$ strongly exposes $B(E)$ at $u$ and $u$ strongly exposes $B\left(E^{*}\right)$ at $u^{*}$, then $\varphi$ is Fréchet differentiable at $x$ with derivative $u^{*}$ and every minimizing sequence for $x$ in $M$ converges to $x-\varphi(x) u$.

ProOF. Since $|\varphi(y)-\varphi(z)| \leq\|y-z\|$ for all $y$ and $z$ we have $1=D_{u} \varphi(x) \leq$ $N_{\varphi}(x) \leq L_{\varphi}(x) \leq 1$ so $D_{u} \varphi(x)=L_{\varphi}(x)=1$ and the norm of $E$ is Fréchet differentiable at $u$ with derivative $u^{*}$ by Proposition 3.1. Now Corollary 2.6 shows that $\varphi$ is Fréchet differentiable at $x$ with derivative $u^{*}$ and the other statement follows from Proposition 3.2.

3.4 COROLlaRY. Suppose that the norms of $E$ and $E^{*}$ are Fréchet differentiable. If $D_{u} \varphi(x)=1$ for some $u \in S(E)$ then $P$ is continuous at $x$.

Proof. By Proposition 3.1, $u$ strongly exposes $B\left(E^{*}\right)$ at a point $u^{*}$ and $u^{*}$ strongly exposes $B(E)$, necessarily at the point $u$. Now Theorem 3.3 shows that every minimizing sequence for $x$ converges to $x-\varphi(x) u$ so $P$ is continuous at $x$ and $P(x)=x-\varphi(x) u$. 
A Banach space $E$ is strictly convex if $S(E)$ contains no line segments and $M$ is a Cebyšev set in $E$ provided every $x \in E$ has a unique nearest point in $M$. We need the following result of Vlasov [6]; see also [3, §4.2].

3.5 PROPOSITION. If $E^{*}$ is strictly convex, then every Čebyšev set in $E$ with continuous metric projection is convex.

Our final result improves Theorem 4.2 of [2].

3.6 THEOREM. Let $M$ be a closed subset of $E$ such that for each $x \in E \backslash M$ there exists $u \in S(E)$ such that $D_{u} \varphi(x)=1$. If the norms of $E$ and $E^{*}$ are Fréchet differentiable, then $M$ is convex.

Proof. From Corollary 3.4 we see that $M$ is a Cebyšev set and $P$ is continuous at each $x \in E \backslash M$. Also $E$ is reflexive $\left[1\right.$, p. 34 , Corollary 1] and $E^{*}$ is strictly convex [1, p. 24, Corollary 1]. Applying Proposition 3.5 we find that $M$ is convex.

\section{REFERENCES}

1. Joseph Diestel, Geometry of Banach spaces-selected topics, Springer Verlag, New York, 1975.

2. Simon Fitzpatrick, Metric projections and the differentiability of distance functions, Bull. Austral. Math. Soc. 22 (1980), 291-312.

3. J. R. Giles, Convex analysis with application in differentiation of convex functions, Pitman, London, 1982.

4. R. R. Phelps, Differentiability of convex functions on Banach spaces, lecture notes from a postgraduate course, University College, London, 1978.

5. V. Smulian, Sur la dérivabilité de la norme dans les espaces de Banach, Rec. Math. [Mat. Sb.] N. S. 9(5) (1941), 545-561.

6. L. P. Vlasov, Almost convexity and Chebyshev sets, Math. Notes. Acad. Sci. USSR 8 (1970), 776-779.

Department of Mathematics and Statistics, University of AuCkLAnd, PriVATE BAG, AUCKLAND, NeW ZeAland 